\title{
PROGRAMA NACIONAL DE PREVENCIÓN DEL SUICIDIO DE CHILE: UNA COMPARACIÓN CON LA EVIDENCIA INTERNACIONAL
}

\author{
CHILEAN NATIONAL SUICIDE PREVENTION PROGRAM: ITS \\ EXAMINATION WITH INTERNATIONAL EVIDENCE
}

\section{Carolina Inostroza Rovegno*}

\begin{abstract}
Resumen
El suicidio es un problema de salud pública mundial que ha sido abordado a nivel internacional con programas nacionales de prevención. El Programa Nacional de Prevención del Suicidio (PNPS) de Chile, es descrito y comparado con la evidencia internacional; la cual señala como componentes efectivos: Programa nacional de prevención, estrategias preventivas específicas a nivel universal, selectivo e indicado. En el análisis se detecta que las medidas planteadas por el PNPS son concordantes con la literatura, pero que su implementación aún es deficitaria en varias de ellas. Son fortalezas: cobertura territorial de salud mental y PNPS; contar con guías clínicas GES y orientaciones técnicas del PNSP; formación de actores clave en los sistemas de salud y educación. Se detallan propuestas de mejora acordes con la evidencia internacional y viables de implementar en Chile. Como elementos centrales a mejorar se cuentan: el aumento del presupuesto otorgado al PNPS y a salud mental en general; favorecer el acceso a tratamiento de salud mental en etapas tempranas del riesgo a personas en situación de vulnerabilidad social; la intervención en crisis, tratamiento y seguimiento de las personas con crisis suicida; la vigilancia epidemiológica de intentos de suicidio y suicidios consumados; y la evaluación científica del programa. Son oportunidades: La existencia de ONGs dedicadas a la prevención del suicidio, y de una academia con experticia en el tema; actores clave cuya integración al PNPS favorecería el logro de este importante objetivo.
\end{abstract}

Palabras clave: Suicidio, prevención, salud mental, basado en evidencia.

\begin{abstract}
Suicide is a global public health problem that has been addressed internationally with national prevention programs. Chilean National Suicide Prevention Program (PNPS) will be described and compared with international evidence. Effective components are: National prevention program, specific preventive strategies at universal, selective, and indicated level. The PNPS is analysed and compared, detecting that the measures proposed by it are consistent with the available evidence, but that its implementation is still deficient in several components. Strengths: territorial coverage of mental health and PNPS; existence of PNSP and GES clinical guidelines; progress in training of key actors in health and education systems. Proposals for improvement are detailed, in accordance with international evidence and feasibility of implementation in Chile. The main elements to be improved include, increasing the budget allocated to the PNPS and mental health; favour access to mental health treatment in early stages of risk for people in social vulnerability; crisis intervention, treatment, and follow-up of people in suicidal crisis; epidemiological surveillance of suicide attempts and completed suicides; and scientific evaluation of the program. Opportunities: The existence of NGOs dedicated to suicide prevention, and academic experts in the field; key actors whose alliance with PNPS would favour the achievement of this important goal.
\end{abstract}

Key words: Suicide, prevention, mental health, evidence based.

Recibido: 16 de septiembre de 2021 /Aceptado: 10 de enero de 2022

\footnotetext{
* Doctora en Psicología, Departamento de Psicología, Facultad de Ciencias Sociales, Universidad de Concepción.Chile Correo: caroinostroza@udec.cl. Código ORCID: http://orcid.org/0000-0001-5415-4169.

La presente publicación corresponde al proyecto VRID Asociativo 218.172.023-10, Universidad de Concepción.
} 


\section{Introducción}

Las muertes por suicidio constituyen un problema que atañe a todos los países, por lo que desde la mitad del siglo pasado se vienen desarrollando programas para su reducción, con énfasis en los países desarrollados. De este modo, hoy en día contamos con evidencia internacional sobre qué elementos son necesarios y efectivos a la hora de implementar un plan para su prevención.

En el caso de Chile, la reducción de las muertes por suicidio es foco de los Planes Nacionales de Salud Mental y Psiquiatría Comunitaria que se desarrollan desde el año 2000; objetivos y estrategias específicos para este fin han sido definidos en un Programa Nacional de Prevención del Suicidio, en adelante PNPS, que fue promulgado en el año 2013.

El objetivo de este artículo es presentar aquellos elementos que han resultado ser más efectivos en el plano internacional para disminuir las tasas de suicidio, los cuales se contrastarán con lo diseñado e implementado por el Ministerio de Salud chileno en la última década. Luego se presentarán sugerencias respecto al camino a seguir en los próximos años.

\section{Desarrollo}

\subsection{Magnitud del Problema}

Las muertes por suicidio son una importante causa de mortalidad. Constituyen la décima causa de muerte para población general en el mundo, y la segunda causa de muerte tanto para los jóvenes entre los 15 y 29 años como para las personas con trastornos mentales (Turecki et al., 2019; Organización Mundial de la Salud, OMS, 2014).

La Organización Mundial de la Salud (OMS, 2014), en su último informe sobre el tema, reporta una tasa de suicidio promedio mundial de 11,4 suicidios por cada 100.000 habitantes, equivalente a 804.000 muertes anuales. Estas tasas reflejan un estancamiento de las alzas reportadas en informes anteriores en la mayoría de los países, y una reducción del suicidio en países que han desarrollado planes integrados de prevención.

En nuestro país el suicidio es la segunda causa de muerte no natural, siendo cercana al número de muertes por accidentes de tránsito, y el doble que los homicidios; es, además, la segunda causa de muerte global en los adolescentes. Las tasas de suicidio de la década del 2000 varían entre 12,1 al 14,7 por 100.000 habitantes (Bustamante et al., 2015; Otzen et al., 2014). En los últimos cinco años reportados, se observa una tendencia a la disminución de las tasas, pasando de 13,1 suicidios por cada 100.000 habitantes en el año 2009 a 10,6 por cada 100.000 habitantes para el 2013. Ese año, el suicidio fue la causa de muerte de 1738 personas en Chile (Hernández, Zitko \& Gómez; 2018). El suicidio tiene un grave impacto en las personas, la familia y la sociedad (OMS, 2014). Se ha descrito que cada muerte por suicidio afecta entre 11 a 115 personas, con distinto grado de cercanía e impacto (Cerel, 2015). Los cercanos afectados por suicidio tienen alto riesgo de desarrollar problemas de salud mental, destacando la propia conducta suicida a nivel de intentos y suicidio consumado (Erlangsen, \& Pitman, 2017; Erlangsen et al., 2017).

\subsection{Evidencia sobre prevención del suicidio a nivel mundial.}

Los planes nacionales de prevención del suicidio surgen en el mundo desarrollado desde la segunda mitad del siglo XX y a medida que han transcurrido las décadas, contamos con abundante evidencia sobre los componentes y planes que tienen mayor efectividad. A continuación, se presentarán las estrategias de prevención del suicidio que cuentan con mayor respaldo de acuerdo con el informe de la Organización Mundial de la salud, 
Prevención del suicidio: un imperativo global (OMS, 2014), los planteamientos de la asociación europea de Prevención del Suicidio (Platt \& Niederkrotenthaler, 2020; Zalsman et al., 2016; Zalsman et al., 2017), y el Centro de Control de Enfermedades de Estados Unidos (CDC, por sus siglas en inglés; Stone et al., 2017):

Plan nacional integrado de prevención del suicidio: Es necesario contar con un plan y estrategia nacional de prevención del suicidio, que sea implementada por un programa o fuerza de tarea separado del programa de salud mental general -aunque alineada con ély con financiamiento propio. Este programa requiere de una evaluación científica continuada con financiamiento público, y necesariamente debe contar con información de tasas anuales de intentos de suicidio y suicidios consumados.

En los siguientes párrafos se presentarán los componentes y medidas que cuentan con mayor evidencia de efectividad (OMS, 2014; Platt \& Niederkrotenthaler, 2020; Stone et al., 2017; Zalsman et al., 2016; Zalsman et al., 2017); también se comentarán aquellas estrategias que se consideran beneficiosas de acuerdo con el criterio de expertos, pero para las cuales no se cuenta aún con suficiente evidencia debido a la complejidad de realizar ensayos clínicos aleatorizados (ECA o RCT por sus siglas en inglés) en esta temática ${ }^{2}$ (ver figura 1$\left.)\right\}$

Figura 1: Estrategias de Prevención del Suicidio basadas en evidencia

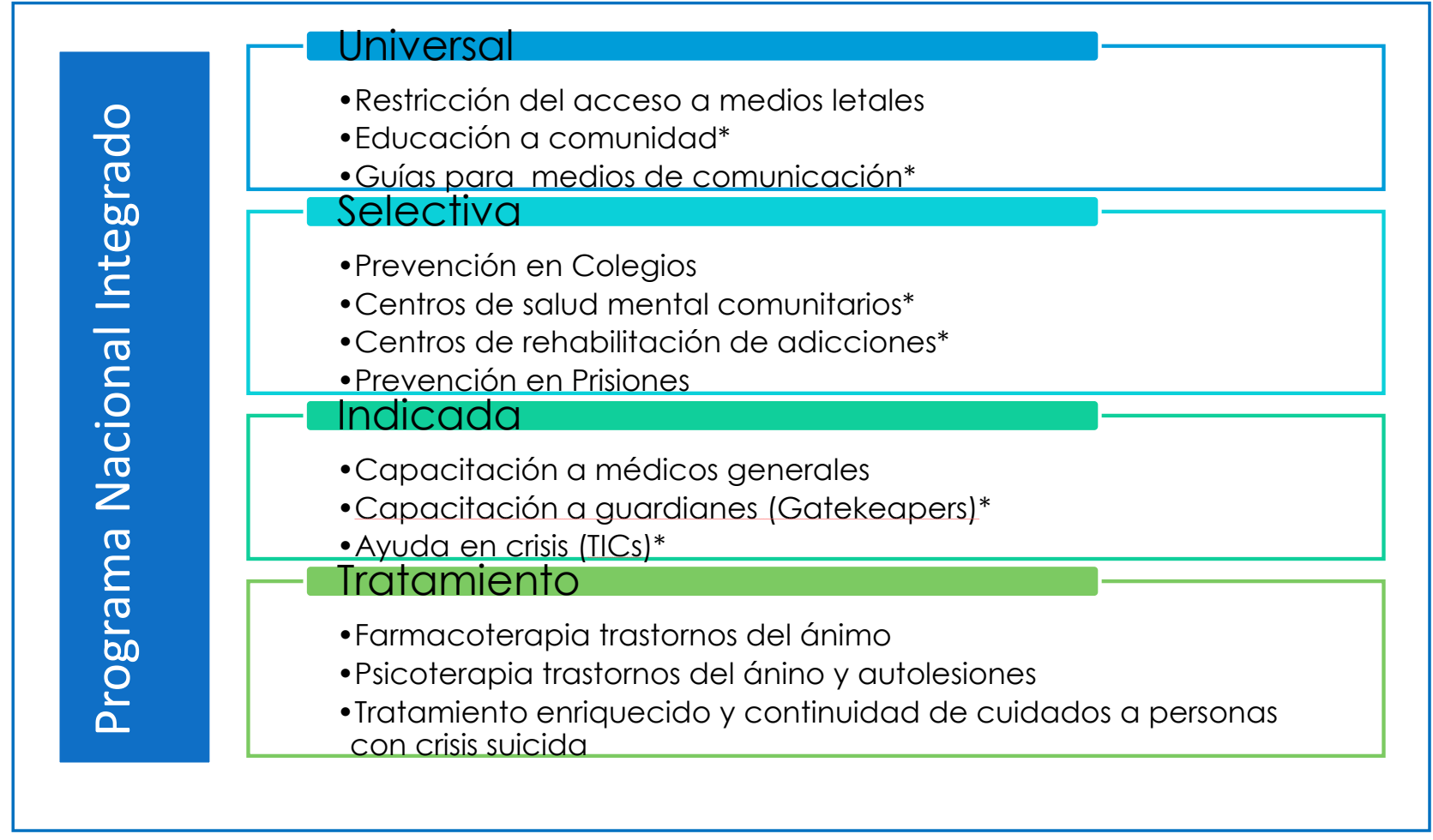

Fuente: Elaboración Propia

*Medidas sin evidencia suficiente a la fecha, pero potencialmente beneficiosas

\footnotetext{
${ }^{2}$ El suicidio es un fenómeno multifactorial y de relativa baja ocurrencia a nivel poblacional. Esto hace que medidas de prevención universal o de salud pública sean muy difíciles de evaluar mediante ensayos clínicos por el tamaño de las muestras requeridas para lograr determinar efectos (potencia del estudio).
} 
Estrategias de prevención universal: corresponde a estrategias útiles para toda la población, tengan o no riesgo suicida.

Restricción del acceso a medios letales: Existen pruebas sólidas respecto de la restricción a las armas de fuego, la reducción de la toxicidad de los pesticidas y el gas domiciliario, el control de los analgésicos y barbitúricos, y el uso de barreras para impedir los saltos de altura. No se cuenta con evidencia sobre programas de restricción del método por ahorcamiento en población general, sólo se sabe de su restricción en las unidades de hospitalización de psiquiatría, donde muestran efectividad en la reducción de las tasas de suicidio de pacientes con alto riesgo.

Campañas de educación a la comunidad: Efectuar campañas por medios de comunicación a la comunidad general, como también a grupos específicos (por ejemplo, adolescentes), sobre el reconocimiento de problemas de salud mental, las formas de pedir ayuda, disminuir el estigma asociado a los trastornos mentales, entre otras; son medidas con un efecto positivo en las actitudes hacia las personas con trastornos mentales y en el aumento en la búsqueda de ayuda; pero aún no se logran suficientes pruebas sobre su aporte a la reducción de las muertes por suicidio.

Guías para la correcta publicación en medios de comunicación: Es sabido que existe el fenómeno de contagio suicida y que éste es favorecido por una mala divulgación en la prensa -y actualmente en las redes sociales- de los casos de suicidio. Las guías de buenas prácticas para los periodistas y medios de comunicación sobre el suicidio incluyen: evitar la descripción detallada y fotografías del caso, no mencionar de los métodos suicidas, evitar el sensacionalismo, romanticismo y divulgación de mitos sobre el suicidio, no publicar casos de suicidio en portada, hacer énfasis en el suicidio como un problema con causas múltiples y nunca debido a un único factor o evento, señalar la posibilidad de prevención y tratamiento, e indicar expresamente líneas y centros de ayuda. De acuerdo con algunos estudios, la correcta aplicación de estas guías reduce las tasas de suicidio, pero en otras investigaciones la evidencia no es concluyente. Se sabe que los mejores efectos se consiguen cuando el diseño e implementación de las buenas prácticas se hace en conjunto con los periodistas.

Prevención selectiva: Se agrupan aquí las buenas prácticas focalizadas en una población de riesgo para el suicidio; como, por ejemplo, los adolescentes, personas con trastornos mentales, entre otros.

Colegios: Incluye una variedad heterogénea de programas desarrollados en el contexto escolar, desde aquellos que buscan generar conciencia sobre la salud mental, otros de apoyo de pares, aplicación de tamizaje, y entrenamiento en habilidades de afrontamiento. Los programas de concientización han demostrado ser útiles para mejorar el conocimiento y las actitudes hacia los trastornos mentales, y en algunos casos para disminuir la ideación o intentos suicidas. Programas enfocados en el desarrollo de habilidades muestran efecto en la reducción de las conductas suicidas en los adolescentes. El entrenamiento de guardianes o gatekeepers (profesores, orientadores, entre otros) no han logrado probar efectividad hasta el momento, aunque se considera una estrategia potencialmente beneficiosa.

Salud Mental en la Comunidad: La intervención a personas con trastornos mentales en la comunidad no necesariamente mejora las tasas de suicidio al compararlo con el tratamiento habitual en centros de psiquiatría, pero sí favorece una mejor aceptación de las intervenciones. Estudios muestran que intervenciones para personas mayores en la comunidad, mediante programas multicomponente con cuidados de acuerdo con niveles de riesgo (stepped-care programs), son útiles para este fin.

Centros de rehabilitación de adicciones: Muy pocos estudios se han realizado para verificar si los tratamientos para adicciones disminuyen el riesgo suicida de este grupo. 
De los pocos que se han conducido hasta ahora, no se han encontrado resultados positivos en conducta suicida, aunque así en la mejoría de otros indicadores de salud mental. Las revisiones consultadas señalan que esta falta evidencia es una brecha crítica en la literatura para la prevención del suicidio, puesto que las personas con abuso de sustancias son un grupo de alto riesgo.

Prisiones: En los últimos años se han desarrollado estudios sobre la efectividad de programas para manejar las conductas suicidas y autolesivas en las prisiones, los cuales han mostrado buenos resultados; estos incluyen trabajo con entrenamiento al personal carcelario, grupos de pares y tratamientos específicos a personas con trastornos mentales encarceladas.

Trabajo: Desafortunadamente, hasta el momento muy pocos programas se han enfocado en la prevención del suicidio en el contexto laboral. Es un área potencialmente útil para desarrollar prevención, pero se requiere de mayores estudios para su consolidación.

Prevención indicada: Serie de programas enfocados en detectar y tratar a aquellas personas que ya están desarrollando algún nivel de conducta suicida no letal (ideación o intento suicida) para evitar que lleguen a morir por suicidio.

Entrenamiento de guardianes o gatekeepers: Consiste el entrenar a grupos específicos de personas que se encuentran en contacto con personas potencialmente en riesgo de desarrollar conductas suicidas, como profesores, sacerdotes, policías, bomberos, farmacéuticos, personal de prisión, militares, entre otros; para que sepan detectar y derivar a tratamiento de forma efectiva. Hasta la fecha, no hay evidencia suficiente para considerar que el entrenamiento en guardianes por sí solo ayuda a reducir las muertes por suicidio, aunque sí se sabe que mejora la concientización sobre salud mental y disminuye el estigma de quienes reciben la capacitación.

Entrenamiento a médicos de atención primaria: Se han desarrollado numerosos programas en diversos países para capacitar a los médicos de atención primaria en detectar y tratar la depresión. La mayoría de los estudios indican que es una medida efectiva, resultando en una mayor detección de la depresión, mayor prescripción de antidepresivos, y a través de ello una reducción de las muertes por suicidio.

Ayuda en crisis: Este grupo de intervenciones comenzaron inicialmente como líneas telefónicas de ayuda, las cuales se han ido complementando en los últimos años con sitios de chats y mensajería a de TICs. Los estudios sobre estas líneas de ayuda muestran que son efectivas en reducir el malestar emocional y desesperanza durante la llamada o conversación en línea, y así reducir el riesgo. Algunos estudios muestran que el efecto puede durar incluso hasta tres meses posterior a la llamada. De todas formas, las revisiones plantean que se requiere de investigaciones con mayor seguimiento y grupos de control. La Organización Mundial de la Salud los considera una buena práctica dentro de un programa multi componente de prevención.

Postvención: Son las actividades desarrolladas con los sobrevivientes de un suicidio (familiares y personas cercanas, compañeros de colegio o trabajo, entre otros) para facilitar su recuperación y prevenir desenlaces negativos, como la conducta suicida. Hasta el momento, los estudios sobre postvención no han tenido resultados positivos en disminuir las conductas suicidas de los grupos de sobrevivientes, por lo que se considera una estrategia sobre la que se requiere mayor investigación.

Tamizaje: Se refiere la aplicación de escalas estandarizadas para determinar la presencia de depresión o riesgo suicida en población general; como, por ejemplo, en colegios, trabajo, o en la comunidad. La evidencia al respecto todavía es controversial, especialmente en los grupos de adolescentes, donde no se conoce de efectos positivos y se ha planteado que pudiese tener riesgos por los falsos positivos que serían referidos a atención de salud mental sin necesitarlo. Donde se considera el tamizaje como una 
práctica positiva es en poblaciones de mayor riesgo; como, por ejemplo, centros de salud primaria o personas con trastornos mentales, consultantes de psiquiatría urgencia; siempre que vaya asociado a un seguimiento posterior de la derivación a atención de salud mental.

Tratamientos farmacológicos: Existen pruebas concluyentes sobre la eficacia del tratamiento con antidepresivos para reducir el riesgo suicida en personas con depresión. Hace algunas décadas existió controversia por el aumento de ideación suicida observada en algunos estudios con adolescentes al iniciar el tratamiento antidepresivo; sin embargo, la evidencia acumulada a través de los años mantiene el efecto positivo del tratamiento, teniendo la consideración de una adecuada supervisión por parte de los padres o tutores. Otro fármaco probado para la reducción del riesgo suicida es el uso del litio por parte de las personas con trastorno bipolar.

Intervenciones psicoterapéuticas: Se han estudiado diversos programas para la reducción de las autolesiones y el riesgo suicida. Dos de ellas, la terapia cognitiva conductual y terapia conductual dialéctica tienen efectos positivos en la reducción de las autolesiones en adultos, especialmente en personas que reciben el diagnóstico de trastorno de personalidad límite. Para el caso de los adolescentes la evidencia es menor, pero positiva para la terapia conductual dialéctica, la terapia de mentalización y la terapia familiar. Es necesario que se desarrollen más estudios en adolescentes, debido a la alta incidencia de autolesión y el riesgo de muerte por suicidio asociado a estas conductas.

En los últimos años se han comenzado a desarrollar intervenciones psicoterapéuticas en formato digital (especialmente de orientación cognitivo conductual), comunidades de grupos de autoayuda en línea, y desarrollo de aplicaciones móviles (apps) para la reducción del riesgo suicida. La evidencia hasta el momento es muy débil como para su recomendación, pero prometedora; ya que se han encontrado resultados positivos en la disminución de la ideación suicida en el corto plazo. La OMS señala que internet y las redes sociales son un escenario potencialmente apropiado para el desarrollo de prevención universal.

Tratamiento enriquecido y continuidad de cuidados: Considera una serie de medidas para las personas que han desarrollado intentos de suicidio o autolesiones repetidas. Dentro de éstas destacan, el seguimiento telefónico y las visitas domiciliarias al alta hospitalaria o de la urgencia, y pases de acceso preferencial a hospitalización en caso de necesidad. La evidencia sobre estas medidas es poco concluyente en los países desarrollados, pero prometedora y positiva en los países de medianos y bajos ingresos como el nuestro.

\subsection{Programa Nacional de Prevención del Suicidio de Chile}

La reducción del suicidio ha formado parte de las metas sanitarias del país durante este siglo (Ministerio de Salud, Chile, 2002; 2010a). Se incluyen acciones para el logro de este objetivo en las distintas versiones del Plan Nacional de Salud Mental chileno (Ministerio de Salud, Chile, 2000; 2017; Minoletti \& Zaccaria, 2005); y en estrategias específicas, como el plan de Garantías Explícitas en Salud (GES) N 15 Esquizofrenia, N³4 Depresión, $N^{\circ} 53$ Consumo perjudicial o dependencia de riesgo bajo a moderado de alcohol y drogas y N ${ }^{\circ} 75$ Trastorno Bipolar (Ministerio de Salud, Chile, s.f).

Pese a estas medidas, la evaluación de los objetivos sanitarios del año 2001 al 2010 (Ministerio de Salud, Chile, 2010b) mostró que, en vez de ocurrir una disminución las tasas de suicidio, estas continuaron subiendo, con peak importante en los años $2007 \mathrm{y}$ 2008 (Bustamante et al., 2015: Otzen et al., 2014). En este contexto, surge el Programa Nacional de Prevención del Suicidio en el año 2008, iniciando de forma piloto en algunas regiones del país, y consolidándose con la Norma General Administrativa 027 "Programa Nacional de Prevención del Suicidio: orientaciones técnicas para su implementación" 
(Ministerio de Salud, MINSAL, 2013), y complementos posteriores (Ministerio de Desarrollo Social y Familia, Chile, 2019; 2020).

En los siguientes apartados se describirán los componentes del PNPS y se comentará su desarrollo:

Instalación de un sistema de estudio de casos: Considera el desarrollo de un sistema de vigilancia epidemiológica de los suicidios consumados y también de los intentos de suicidio. Esta función recae en las SEREMI de salud, las cuales han desarrollado sus sistemas de vigilancia de forma autónoma del nivel central, y con distintos niveles de implementación en cada región. Las estadísticas de muertes por suicidio son desarrolladas el Departamento de Estadísticas e Información de Salud (DEIS), el cual informa datos anuales de mortalidad por región y comuna para todo el país ${ }^{3}$, dotándonos de información de calidad y útil para la prevención. Distinto es el caso de los intentos de suicidio; hasta la fecha, no se ha logrado una definición de caso de intento de suicidio común a todas las regiones del país, tampoco se ha conseguido que el intento de suicidio sea tratado como una enfermedad de notificación obligatoria (ENO), como es el caso de otras enfermedades de interés epidemiológico para las cuales tenemos una vigilancia reconocida a nivel mundial (Ministerio de Salud, Chile, 2002; s.f.).

Otra medida propuesta para este componente es el desarrollo de autopsias psicológicas de los casos de muerte por suicidio. Si bien, la información obtenida por esta metodología es profunda y útil para la prevención (Cañón et al., 2016), su implementación es muy costosa y difícil de ejecutar; por lo que, a la fecha, sólo contamos con una publicación que reporta resultados de 30 autopsias psicológicas, desarrollada por el doctor Alejandro Gómez y equipos de la región de Coquimbo (Gómez et al, 2014).

Implementación de un Plan Regional Intersectorial de Prevención del Suicidio: Se refiere a la conformación de una mesa intersectorial para la generación de un plan regional de prevención del suicidio. Deberán formar parte de ella los sectores de salud, educación, carabineros, bomberos, organizaciones civiles, entre otras; y contar con canales de comunicación y reuniones con una frecuencia adecuada para implementación de estrategias. Dentro de las acciones propuestas se incluyen: medidas para la restricción a medios letales, campañas de fomento de factores protectores, disminución de la estigmatización y favorecer la búsqueda de ayuda.

Esta es una estrategia con una lógica adecuada de acuerdo con los modelos de salud pública y salud mental comunitaria. A la fecha, se han desarrollado planes y mesas regionales en cada una de las regiones del país; pero, su nivel de ejecución efectiva es muy dispar; con tres regiones con un buen nivel de funcionamiento -las tres regiones que han recibido financiamiento- - y las restantes con amplias diferencias en su implementación (Ministerio de Desarrollo Social y Familia, Chile, 2019). Si bien, la creación de planes regionales es coherente con el reconocimiento de que el contexto ambiental y sociocultural es relevante para la prevención del suicidio (Turecki et al., 2019); y, por lo tanto, está es una estrategia que se debe mantener, es necesario homologar hacia arriba el nivel de funcionamiento de mesas y planes regionales.

Fortalecimiento de las competencias de los profesionales de la salud: De forma concordante con las pruebas de que la capacitación a médicos de atención primaria es una de las medidas preventivas efectivas para la prevención del suicidio, el Plan Nacional considera la capacitación a funcionarios de salud, con énfasis en la atención primaria y los servicios de urgencia. Es sabido que estos entrenamientos son uno de los componentes que se ha ejecutado de forma estable en los últimos años, aunque nuevamente con mejor

\footnotetext{
${ }^{3}$ Sitio web https://deis.minsal.cl/\#datosabiertos
} 
desarrollo en algunas las regiones que en otras. La reformulación del PNPS, en su componente financiado por el Ministerio de Desarrollo Social y Familia (2020) destina fondos a la ejecución de esta capacitación y la del apartado siguiente.

Incorporación de programas preventivos en los establecimientos educacionales: Forma parte de este ámbito, el desarrollo de planes preventivos en las comunidades escolares, y su articulación intersectorial, particularmente con la atención primaria de salud. El trabajo en las comisiones mixtas de salud y educación (COMSE) con foco promocional y preventivo es una estrategia que forma parte de la atención primaria de salud chilena y que da sustento a este componente.

En el año 2019 se publicaron las "Recomendaciones para la prevención de la conducta suicida en establecimientos educacionales" (Ministerio de Salud, Chile, 2019), fruto de trabajo colaborativo entre los equipos de salud y expertos nacionales. Las orientaciones de esta guía van de la mano con la literatura sobre prevención del suicidio en contextos escolares. Hasta el momento se encuentra en etapa de implementación inicial, ya que requiere de una acción intersectorial desde los Ministerios de Educación y Salud hacia los territorios. Cómo se mencionó, la reformulación del PNPS (Ministerio de Desarrollo Social y Familia, 2020) incluye recursos para la formación de personal de las comunidades escolares.

Desarrollo de un sistema de ayuda en situaciones de crisis: Los sistemas de atención de urgencias y crisis suicida son un componente básico de cualquier programa preventivo. En el PNPS se propone la existencia de una línea telefónica de ayuda en crisis, una página de internet, un protocolo de intervención en crisis y un sistema de seguimiento de los casos que han realizado intentos de suicidio.

Hasta el momento, se ha instalado una línea de atención telefónica en el sistema Fono Salud Responde, el cual cuenta con atención de psicólogos 24 horas para orientación de los casos en crisis. Estas ayudas son complementadas con diversas organizaciones no gubernamentales (en adelante, ONGs) que tienen sistemas de ayuda por salas de chat y mensajería, más atingentes a la cultura juvenil y de redes sociales (en adelante, RRSS) que se ha masificado en los últimos años. Aunque no se han desarrollado protocolos regionales de intervención en crisis suicida, las orientaciones técnicas del PNPS, y la implementación de la psiquiatría de urgencia en los hospitales de mayor complejidad, favorecen las acciones de los equipos de salud ante las crisis suicidas. Queda pendiente un sistema de seguimiento y continuidad de cuidados de las personas que realizan un intento de suicidio y que son atendidas en el sistema de urgencias (urgencia hospitalaria, SARS/SAPU). Este último componente es de suma relevancia, ya que se sabe que el principal factor de riesgo para los suicidios consumados, son los intentos previos, cuyo riesgo es mayor en el primer año siguiente a un intento (Choi et al., 2012; Christiansen \& Jensen, 2007).

Apoyo para una correcta cobertura mediática y refuerzo medios de comunicación en la prevención del suicidio: La relevancia de los medios de comunicación en la prevención del suicidio actúa por lo menos de dos formas. Por un lado, se debe prevenir el efecto de contagio suicida a través de una correcta información sobre el suicidio; y por otro, pueden ser ejercer un rol promotor de la salud mental, disminución del estigma y favorecer la búsqueda de ayuda de las personas en riesgo. Para que esto ocurra, el PNPS señala que se debe realizar un trabajo de colaboración y capacitación con los periodistas y los medios de comunicación, incluyendo el desarrollo de guías para una correcta cobertura mediática. Este es uno de los componentes con un bajo nivel de desarrollo inicial, pero en el que se ha visto progresos en los últimos años. 


\section{Propuestas de Mejora}

En esta sección se reflexionará en torno a estrategias que potenciarían el PNPS, tomando en cuenta cómo fue diseñado y cómo ha sido su implementación hasta la fecha. Este análisis se realizará tomando como referencia las medidas efectivas para la prevención del suicidio expuestas previamente (Platt \& Niederkrotenthaler, 2020; Zalsman et al., 2017; Zalsman et al., 2017).

\subsection{Plan nacional integrado de prevención del suicidio}

El PNPS de Chile cuenta con algunos elementos señalados como eficaces: Un plan normado para todo el territorio nacional, que integra acciones de los distintos niveles preventivos, e incluye unidades territoriales regionales coordinadas por el nivel central. Una posible debilidad, de acuerdo con la literatura, es que el PNPS es un elemento más del programa de salud mental, y no un ente independiente cómo proponen las experiencias internacionales exitosas. Esto toma relevancia considerando que varios componentes del PNPS necesitan de un trabajo intersectorial que implica coordinaciones entre ministerios; por lo cual, se requeriría de una reestructuración para que el PNPS tenga un nivel equivalente en la jerarquía gubernamental al Programa de Salud Mental. Lo anterior se relaciona con el escaso presupuesto que ha tenido hasta el momento el PNPS, especialmente si consideramos que el suicidio es una de las causas de muerte principales en los jóvenes, y la segunda causa de muerte no natural (Turecki et al., 2019; OMS, 2014). Con relación a la vigilancia epidemiológica, una fortaleza en nuestro país es la calidad del registro epidemiológico de los casos de muertes por suicidio, y una debilidad factible de mejorar, es lograr la vigilancia epidemiológica de los intentos de suicidio a través de las enfermedades de notificación obligatoria (ENO). Para que el registro epidemiológico de intentos y suicidios consumados sea efectivo como medida preventiva, requiere ser informado a los equipos sectoriales de salud mental; de esta forma podrán ejecutarse medidas correctivas. En relación con los suicidios consumados y las autopsias psicológicas; el tiempo ha demostrado que estas son de difícil ejecución. Una alternativa posible de implementar es el análisis de los protocolos de autopsia realizados por los servicios médicos legales (SML), a través de los cuales se puede obtener amplia información sobre las características de las personas que mueren por suicidio (Baader et al., 2011, Inostroza et al., en preparación).

Finalmente, es necesario desarrollar una evaluación científica del programa y de sus componentes. Nuestro país cuenta con expertos en prevención del suicidio y evaluación de programas en la Academia que han colaborado previamente con el sector salud (Gómez, Baader et al., 2011; Gómez et al., 2014; Saldivia, Grandón, Albornoz \& Toro, 2008, entre otros), lo que facilitaría la ejecución de este necesario análisis científico.

\section{Estrategias de prevención universal:}

Restricción del acceso a medios letales: El principal método suicida en Chile es el ahorcamiento (Bustamante et al., 2015), por lo que es recomendable incluir acciones que apunten a este método. Si bien, es poco factible impedir el acceso universal a formas de ahorcamiento como ocurre con otros métodos (por ejemplo, armas de fuego), es necesario educar de forma específica sobre el riesgo por este método en las capacitaciones a los profesionales de la salud, de educación y agentes comunitarios; e incluir medidas para su restricción en los Guías Clínicas y protocolos de intervención de los trastornos mentales asociados al suicidio que forman parte del plan GES (Depresión, Trastorno Bipolar, Esquizofrenia, Consumo problemático de alcohol y otras drogas). Lo anterior no excluye que deben mantenerse los esfuerzos en torno a la restricción de los otros medios letales, 
tales como el control de armas, el acceso a medicamentos potencialmente letales, el cierre del acceso a lugares de altura, entre otros.

Campañas de educación a la comunidad: En los últimos años, el PNPS ha desarrollado guías educativas para la prevención del suicidio en población de estudiantes universitarios, personas mayores, y contexto COVID-19. Considerando el impacto a todo nivel de las redes sociales, $R R S S$, también se vuelve relevante monitorear la comunicación incorrecta de contenidos suicidas, y utilizar las RRSS como un canal de promoción y educación en salud mental. Para esto último, el PNPS podría asociarse a las ONGs que participan de la prevención del suicidio (Fundaciones Antonia, José Ignacio, Katty Summer, Míranos, Todo Mejora, Para la Confianza, entre otras ${ }^{4}$ ). Una acción posible, es el desarrollo de una nueva mesa -equivalente a las mesas regionales- para el contexto digital y de RRSS, donde dichas ONGs corresponderían al intersector.

Guías para la correcta publicación en medios de comunicación: Con respecto a los medios de prensa, es necesario elaborar guías, profundizar las capacitaciones, y mantener un monitoreo y trabajo colaborativo con los periodistas y medios de comunicación. Las acciones en torno a una correcta comunicación sobre el suicidio deben ir de la mano de campañas de educación en salud mental y combate del estigma hacia las personas con trastornos mentales. En nuestro país existen equipos que se encuentran trabajando en estas temáticas (Grandon et al., 2019; Macaya \& Vicente, 2019).

\section{Prevención selectiva:}

Colegios: Como se mencionó, la Guía de prevención del suicidio para establecimientos educacionales (Ministerio de Salud, 2019) comprende los elementos adecuados de este componente preventivo. El desafío es concretar su implementación a escala nacional, lo cual requerirá de una colaboración planificada entre los Ministerios de Educación y Salud para que las orientaciones técnicas se logren diseminar de forma efectiva hacia los territorios. Un pilar para ello es la capacitación de actores relevantes en cada establecimiento; profesores, equipos psicosociales y de convivencia escolar. En este sentido, la inclusión de una partida presupuestaria para este fin en el año 2020 es una medida que debe sostenerse en el tiempo.

Salud Mental en la Comunidad: Chile tiene implementado hace ya dos décadas un modelo de salud mental y psiquiatría comunitaria (Ministerio de Salud, 2000; 2017) que favorece la aplicación de un PNPS. Un punto clave a mejorar es el acceso real de las personas afectadas por trastornos mentales con riesgo suicida -incluido el abuso de sustancias- a tratamiento. Sólo un 36\% de las personas que se suicidan en Chile se encontraba en tratamiento de salud mental (Gómez et al., 2014), mientras que un $90 \%$ de quienes mueren por suicidio cumplen criterios para un trastorno mental (Turecki et al., 2019), y un 56,7\% se encontraba bajo los efectos del alcohol u otras drogas al momento de su muerte (Inostroza et al., en preparación).

Al mismo tiempo, sabemos que el suicidio en Chile se asocia a una mayor vulnerabilidad y exclusión social (Duarte, 2008), por lo que un programa de prevención del suicidio debería incluir estrategias para poblaciones de riesgo específicas, como son personas con adicciones y problemas con la justicia. En ambos casos se requiere de trabajo no sólo intersectorial, sino que interministerial para lograr la articulación con programas existentes del Servicio Nacional para la Prevención y Rehabilitación del Consumo de Drogas y Alcohol (SENDA) y el Ministerio de Justicia.

Del mismo modo, somos conscientes de que nuestro país, si bien tiene un buen nivel de salud pública a nivel latinoamericano, al mismo tiempo presenta grandes problemas de

\footnotetext{
${ }^{4}$ https://repschile.org/ para un listado de ONGs de prevención del suicidio en Chile.
} 
inequidad, especialmente en salud mental (Araya, Rojas, Fritsch, Frank \& Lewis, 2006; Frenz, Delgado, Kaufman \& Harper, 2014; Gatica-Saavedra, Vicente, Vicente \& Rubí, 2020); para cuya mejora es fundamental un aumento del presupuesto (Errázuriz, Valdés, Vöhringer \& Calvo, 2015). Junto con el aumento presupuestario, se requiere trabajar en la implementación de una política de salud mental con un énfasis en promoción y prevención, que incluya como eje la participación ciudadana, y evite de forma activa la psicopatologización y estigmatizacíón del sufrimiento (Cova, Grandon, Saldivia, Inostroza \& Novoa, 2019).

\section{Prevención indicada:}

Entrenamiento de guardianes (gatekeepers) y médicos de atención primaria: Esta estrategia se encuentra en la reformulación del PNPS del año 2020 (Ministerio de Desarrollo Social y Familia, 2020) que contempla la capacitación a profesionales de la salud, a profesores y otros estamentos educativos, y la formación de agentes comunitarios. El enfoque en estos tres grupos se considera apropiado, y se sugiere un aumento presupuestario para lograr una cobertura adecuada de guardianes con entrenamiento en la detección y asistencia en la búsqueda de ayuda de personas con riesgo suicida.

Ayuda en crisis: La incorporación de profesionales de salud mental en el Sistema de Fono Salud Responde es un avance en la instalación de un sistema de ayuda en crisis. Sin embargo, como en otras medidas, su cobertura real y reconocimiento por parte de la comunidad es aún insuficiente. Sería recomendable que este sistema estuviera anclado al número de urgencias 131, o a otro número propio más simple (ejemplos son el Fono Familia 145, Fono Mujer 1455, entre otros). Aunque esto pueda parecer nimio, debemos tener presente que una persona en crisis suicida tiene temporalmente disminuidas las capacidades cognitivas para la resolución de problemas y búsqueda de ayuda (Wenzel \& Beck, 2008), por lo que el acceso directo a ayuda a través de un número telefónico corto y recordable, es una medida que puede marcar una diferencia y posibilitar un rescate. Asimismo, debería existir una línea de chat operativa propia del PNPS, sumada a una acción coordinada con las salas de chat de las ONGs que han sido pioneras en este formato de intervención preventiva.

El acceso a un sistema de ayuda telefónica o en línea es el primer paso, el cual debe estar coordinado de forma eficaz con la respuesta de ambulancias, urgencias, y en algunos casos carabineros o bomberos. Por este motivo, es necesario contar con un sistema de intervención en crisis a través de ambulancias propias, con equipos preparados para el rescate de personas en una crisis suicida; y la capacitación permanente de todo el equipo de rescate de urgencias. Lo anterior debe complementarse con la formación de carabineros y bomberos para que las acciones de rescate puedan ser efectivas, por un lado, y al mismo tiempo, respetar los derechos de las personas con trastornos mentales.

Postvención: Considerando que aún no se cuenta con suficiente evidencia sobre cómo realizar una postvención efectiva hacia los sobrevivientes de suicidio, no es posible dar una sugerencia en este ámbito; más allá de la correcta aplicación del modelo de salud mental comunitaria territorial. En este caso, los llamados a realizar las acciones postventivas serían los equipos de salud de atención primaria (APS) con consultoría por parte de los centros de salud mental especializados (COSAM o unidades de psiquiatría ambulatoria).

Tamizaje: Como se señaló, el tamizaje para detectar personas con depresión o riesgo suicida sigue siendo una medida controversial. Al respecto, parece tener mayor sentido la búsqueda activa de casos dentro de medidas integrales de salud pública, como son el Examen de Medicina Preventiva realizado por la atención primaria de salud (Ministerio 
de Salud, 2013); el cual cuenta con protocolos de evaluación específica para los adolescentes, los adultos y las personas mayores. Parece ser que, más que el tamizaje, la forma de facilitar el acceso a tratamiento de las personas que tienen riesgo suicida, es contar con una salud mental comunitaria bien implementada, sin barreras de acceso a las personas en situación de vulnerabilidad social, y que combate el estigma en salud mental. Tratamientos farmacológicos e intervenciones psicoterapéuticas: Chile cuenta con buenos programas de intervención y guías clínicas GES para los trastornos mentales que aumentan el riesgo suicida: depresión, trastorno bipolar, abuso de alcohol y otras drogas, y esquizofrenia. Se considera pertinente, su complemento con el desarrollo de guías para otras patologías asociadas a mayor riesgo suicida, como los trastornos de personalidad graves y los trastornos de la conducta alimentaria, entre otros (Turecki et al., 2019). Como hemos venido comentando, las medidas necesarias de aplicar por parte del programa de salud mental en nuestro país tienen que ver con la equidad en el acceso, la cobertura y la oportunidad del tratamiento.

Tratamiento enriquecido y continuidad de cuidados: Este punto comprende la prevención indicada del suicidio en su sentido más estricto. La evidencia es contundente respecto a qué quienes tienen mayor riesgo de morir por suicidio son quienes han realizado un intento para acabar con su vida, especialmente en el primer año siguiente a ese evento. El tratamiento enriquecido para personas con riesgo suicida y la continuidad de cuidados posterior a la descarga de la urgencia o de una hospitalización en psiquiatría debería estar normado en Chile por una guía clínica específica, cómo son las guías clínicas GES. Debemos tener presente que las revisiones sobre prevención del suicidio nos muestran que estas acciones son especialmente eficaces en países de bajos y medianos ingresos como el nuestro (Platt \& Niederkrotenthaler, 2020). Para que esto sea operativo, debe estár integrado con una vigilancia epidemiológica de los intentos de suicidio, cuya información esté disponible para todos los equipos de una red de salud mental comunitaria, de forma tal que puedan implementar acciones de forma coordinada entre sí.

\section{Conclusiones}

Luego de comparar el Programa Nacional de Prevención del Suicidio (PNPS) de Chile con la literatura internacional, se observa que las medidas planteadas por este son concordantes con las estrategias probadamente efectivas y las recomendaciones de los expertos. Si evaluamos la implementación del PNPS tomando en consideración los informes existentes, la experiencia de los equipos de salud y de las ONGs asociadas, detectamos que varias de las estrategias para la prevención del suicidio requieren de mayor desarrollo y, especialmente, de una ejecución con un nivel equivalente en los distintos territorios del país. Esto se comprende por el bajo presupuesto que han tenido hasta la fecha tanto el PNPS como el programa de salud mental.

Chile cuenta con una salud pública sólida y un modelo de salud mental comunitaria instalado; ambos elementos constituyen una base apropiada para la implementación de un PNPS exitoso. Como principales fortalezas destacamos: La cobertura nacional efectiva del programa de salud mental y del PNPS; el contar con guías clínicas GES y orientaciones técnicas del PNSP que permitirían lograr intervenciones con calidad apropiada y equivalente; los avances obtenidos en los últimos años en la formación de actores clave en los sistemas de salud y educación. Como elementos centrales a mejorar se proponen: el aumento del presupuesto otorgado al PNPS y salud mental en general; generar medidas para favorecer el acceso a tratamiento de salud mental en etapas tempranas del riesgo a personas en situación de vulnerabilidad social; el desarrollo de protocolos de intervención en crisis, tratamiento y continuidad de cuidados de las 
personas con crisis suicida; lograr la vigilancia epidemiológica de intentos de suicidio a través de ENO, y alimentar con esta información -y los casos de suicidios consumadosa los equipos territoriales de salud mental; y la evaluación científica del programa.

En nuestro país existen dos actores claves que pueden integrarse a esta iniciativa, ONGs dedicadas a la prevención del suicidio validadas por la población, y un cuerpo de investigadores en salud mental y expertos en prevención del suicidio. Creemos que una alianza del PNPS con estos grupos, sería un elemento potenciador para el éxito del programa.

\section{Referencias}

Araya, R., Rojas, G., Fritsch, R., Frank, R., Lewis, G. (2006) "Inequities in Mental Health Care After Health Care System Reform in Chile", American Journal of Public Health 96, (1),109-113. https://doi.org/10.2105/AJPH.2004.055715

Arensman, E., Scott, V., De Leo, D., \& Pirkis, J. (2020). Suicide and suicide prevention from a global perspective. Crisis.

Baader, T., Behne, P., Molina, J. L., Gacitúa, L., Yáñez, L., Urra, E., \& Millán, R. (2011). ¿Está cambiando la prevalencia de los suicidios y sus características en la población chilena?: análisis de las tasas de suicidios y sus características sociodemográficas, ocurridas en la provincia de Valdivia, actual Región de Los Ríos, entre los años 1996 a 2008. Revista chilena de neuro-psiquiatría, 49(3), 273-282.

Bustamante, F., Ramirez, V., Urquidi, C., Bustos, V., Yaseen, Z. \& Galynker, I. (2015). Trends and Most Frequent Methods of Suicide in Chile Between 2001-2010. Crisis 37(1): 21-30.

Cañón, S. C., Palacios, S. G., Díaz, J. E., Gonzales, E. B., Uribe, V. P., \& Lopez, M. A. A. (2016). Autopsia psicológica: una herramienta útil en la caracterización del suicidio. Archivos de Medicina (Col), 16(2), 410-421.

Cerel, J. (2015, June). Responding to grief, trauma, and distress after suicide: U.S. national guidelines. Presentation at the conference of International Association of Suicide Prevention, Montreal, QC

Choi, J. W., Park, S., Yi, K. K., \& Hong, J. P. (2012). Suicide mortality of suicide attempt patients discharged from emergency room, nonsuicidal psychiatric patients discharged from emergency room, admitted suicide attempt patients, and admitted nonsuicidal psychiatric patients. Suicide and Life-Threatening Behavior, 42(3), 235-243.Duarte, D. (2008). El suicidio en Chile: Un signo de exclusión. Santiago: Universitaria.

Christiansen, E., \& Frank Jensen, B. (2007). Risk of repetition of suicide attempt, suicide or all deaths after an episode of attempted suicide: a register-based survival analysis. Australian \& New Zealand Journal of Psychiatry, 41(3), 257-265.

Cova, F. C., Grandon, P. G., Saldivia, S. S., Inostroza, C. I., \& Novoa, C. N. (2019). Promoción y prevención en salud mental: ¿Esperanza fundada, vana ilusión o contrabando psicopatologizador? Papeles del psicólogo, 40(3), 211-216.

Duarte, D. (2008). El suicidio en Chile: Un signo de exclusión. Santiago: Universitaria. Errázuriz, P., Valdés, C., Vöhringer, P. A., \& Calvo, E. (2015). Financiamiento de la salud mental en Chile: una deuda pendiente. Revista Médica de Chile, 143(9), 1179-1186. Erlangsen, A., \& Pitman, A. (2017). Effects of suicide bereavement on mental and physical health.

Erlangsen, A., Runeson, B., Bolton, J. M., Wilcox, H. C., Forman, J. L., Krogh, J., Conwell, Y. (2017). Association between spousal suicide and mental, physical, and social 
health outcomes: A longitudinal and nationwide register-based study. JAMA Psychiatry, 74(5), 456-464.

Frenz, P; Delgado, I.; Kaufman, U.S. \& Harper, S. (2014). Achieving effective universal health coverage with equity: evidence from Chile, Health Policy and Planning, 29 (6) 717-731, https://doi.org/10.1093/heapol/czt054

Gatica-Saavedra, M., Vicente, B., \& Rubí, P. (2020). Plan nacional de salud mental. Reflexiones en torno a la implementación del modelo de psiquiatría comunitaria en Chile. Revista médica de Chile, 148(4), 500-505.

Gómez Ch, A., Opazo, R., Levi, R., Gómez Ch, M. S., Ibáñez, C., \& Núñez, C. (2014). Autopsias psicológicas de treinta suicidios en la IV Región de Chile. Revista Chilena de Neuro-Psiquiatría, 52(1), 9-19. doi: 10.4067/S0717-92272014000100002

Grandón, P., Saldivia, S., Vaccari, P., Ramirez-Vielma, R., Victoriano, V., Zambrano, C., ... \& Cova, F. (2019). An integrative program to reduce stigma in primary healthcare workers toward people with diagnosis of severe mental disorders: a protocol for a randomized controlled trial. Frontiers in Psychiatry, 10, 110.

Hernández, Zitko \& Gómez (2018). Epidemiología en A. Gómez; H. Silva \& R. Amon. El Suicidio: Teoría y Clínica (pp. 22-27). Santiago: Mediterráneo.

Macaya Sandoval, X., \& Vicente Parada, B. (2019). Alfabetización en salud mental para disminuir la brecha de atención en población adolescente escolarizada. Gaceta Médica Espirituana, 21(1), 70-82.

Méndez, C.A., Greer, S.L. \& McKee, M. The 2019 crisis in Chile: fundamental change needed, not just technical fixes to the health system. J Public Health Pol 41, 535-543 (2020). https://doi.org/10.1057/s41271-020-00241-2

Ministerio de Desarrollo Social y Familia, Chile (2019). Monitoreo y seguimiento oferta pública 2019: Programa Nacional de Prevención del Suicidio.

Ministerio de Desarrollo Social y Familia, Chile (2020). Monitoreo y seguimiento oferta pública 2020: Programa Nacional de Prevención del Suicidio.

Ministerio de Salud, Chile (2013). Guía Clínica Auge Examen de Medicina Preventiva [Internet]. Subsecretaría de Salud Pública. Serie Guías Clínicas Minsal. $\quad$ https://diprece.minsal.cl/wrdprss_minsal/wpcontent/uploads/2014/09/GPC-Medicina-Preventiva.pdf Consultado septiembre 10, 2021 Ministerio de Salud, Chile (2002a). Los objetivos sanitarios para la década 2000-2010. Disponible en https://www.minsal.cl/portal/url/item/6bdb73323d19be93e04001011f013325.pdf

Consultado septiembre 01, 2021

Ministerio de Salud, Chile (2002b). Norma Técnica No 55 de Vigilancia de enfermedades transmisibles. http://epi.minsal.cl/wp-content/uploads/2016/03/enftransmisibles.pdf

Ministerio de Salud, Chile (2010a). Estrategia Nacional de Salud para el cumplimiento de los Objetivos Sanitarios de la década 2011-2020. Disponible en http://www.minsal.cl/portal/url/item/c4034eddbc96ca6de0400101640159b8.pdf

Consultado septiembre 03, 2021

Ministerio de Salud, Chile (2010b). Los Objetivos Sanitarios para la Década 2000-2010. Evaluación de final del período, Grado de cumplimiento de los objetivos de Impacto. Disponible en https://www.minsal.cl/portal/url/item/94c89f56c4e270b0e04001011e011c9c.pdf

Consultado septiembre 08, 2021

Ministerio de Salud, Chile (s.f.a). Guías Clínicas AUGE. Sitio Web: Sitio Web: https://diprece.minsal .cl/le-informamos/auge/acceso-guias-clinicas/guias-clinicasauge/ Consultado septiembre 05, 2021. 
Ministerio de Salud, Chile (s.f.b). Anexo 4: Vigilancia de Enfermedades Transmisibles de Notificación Obligatoria https://www.minsal.cl/wpcontent/uploads/2021/09/ANEXO-04-2022.pdf

Minoletti, A., \& Zaccaria, A. (2005). Plan Nacional de Salud Mental en Chile: 10 años de experiencia. Revista Panamericana de Salud Pública, 18, 346-358.

Nock, M., Borges, G., Bromet, E., Cha, C., Kessler, R. \& Lee, S. (2008). Suicide and suicidal behavior. Epidemiological Review, 30(1), 133-154. doi: 10.1093/epirev/mxn002

Organización Mundial de la Salud. (2014). Prevención del Suicidio: Un imperativo global. Luxemburgo: Organización Mundial de la Salud. Recuperado de https://apps.who.int/iris/bitstream/handle/10665/136083/9789275318508_spa.pdf?seque nce $=1 \&$ isAllowed $=y$

Otzen, T., Sanhueza, A., Manterola, C. \& Escamilla-Cejudo, J. (2014). Mortalidad por suicidio en Chile: tendencias en los años 1998-2011. Rev Med Chile 2014; 142(3): 305313.

Platt, S., \& Niederkrotenthaler, T. (2020). Suicide prevention programs. Crisis.

Saldivia, S., Grandón, P., Albornoz, E., \& Toro, A. (2008). Estudio de servicio de salud mental en el sistema público de salud de Chile. Informe final., Universidad de Concepción, Editor.

Stone, D.M., Holland, K.M., Bartholow, B., Crosby, A.E., Davis, S., and Wilkins, N. (2017). Preventing Suicide: A Technical Package of Policies, Programs, and Practices. Atlanta, GA: National Center for Injury Prevention and Control, Centers for Disease Control and Prevention.

Turecki, G., Brent, D. A., Gunnell, D., O’Connor, R. C., Oquendo, M. A., Pirkis, J., \& Stanley, B. H. (2019). Suicide and suicide risk. Nature reviews Disease primers, 5(1), 122.

Wasserman, D., Rihmer, Z., Rujescu, D., Sarchiapone, M., Sokolowski, M., Titelman, D., ...Carli, V. (2012). The European Psychiatric Association (EPA) guidance on suicide treatment and prevention. European Psychiatry, 27(2), 129-141. Doi: 10.1016/j.eurpsy.2011.06.003.

Wenzel, A., \& Beck, A. T. (2008). A cognitive model of suicidal behavior: Theory and treatment. Applied and Preventive Psychology, 12(4), 189-201.

Zalsman, G., Hawton, K., Wasserman, D., van Heeringen, K., Arensman, E., Sarchiapone, M., ... \& Zohar, J. (2017). Evidence-based national suicide prevention taskforce in Europe: a consensus position paper. European Neuropsychopharmacology, 27(4).

Zalsman, G., Hawton, K., Wasserman, D., van Heeringen, K., Arensman, E., Sarchiapone, M., ... \& Zohar, J. (2016). Suicide prevention strategies revisited: 10-year systematic review. The Lancet Psychiatry, 3(7), 646-659. 